\title{
Eye-tracking and artificial intelligence to enhance motivation and learning
}

\author{
Kshitij Sharma ${ }^{1 *}$, Michail Giannakos ${ }^{1}$ and Pierre Dillenbourg ${ }^{2}$
}

\author{
* Correspondence: kshitij.sharma@ \\ ntnu.no \\ ${ }^{1}$ Department of Computer Science, \\ Norwegian University of Science \\ and Technology, Trondheim, \\ Norway \\ Full list of author information is \\ available at the end of the article
}

\begin{abstract}
The interaction with the various learners in a Massive Open Online Course (MOOC) is often complex. Contemporary MOOC learning analytics relate with click-streams, keystrokes and other user-input variables. Such variables however, do not always capture users' learning and behavior (e.g., passive video watching). In this paper, we present a study with 40 students who watched a MOOC lecture while their eyemovements were being recorded. We then proposed a method to define stimulibased gaze variables that can be used for any kind of stimulus. The proposed stimuli-based gaze variables indicate students' content-coverage (in space and time) and reading processes (area of interest based variables) and attention (i.e., with-meness), at the perceptual (following teacher's deictic acts) and conceptual levels (following teacher discourse). In our experiment, we identified a significant mediation effect of the content coverage, reading patterns and the two levels of with-me-ness on the relation between students' motivation and their learning performance. Such variables enable common measurements for the different kind of stimuli present in distinct MOOCs. Our long-term goal is to create student profiles based on their performance and learning strategy using stimuli-based gaze variables and to provide students gaze-aware feedback to improve overall learning process. One key ingredient in the process of achieving a high level of adaptation in providing gazeaware feedback to the students is to use Artificial Intelligence (Al) algorithms for prediction of student performance from their behaviour. In this contribution, we also present a method combining state-of-the-art Al technique with the eye-tracking data to predict student performance. The results show that the student performance can be predicted with an error of less than $5 \%$.
\end{abstract}

Keywords: Eye-tracking, Motivation, Learning, MOOCs, Video based learning, Multimodal analytics, Massive open online courses, Deep learning

\section{Springer Open}

\section{Introduction}

We present a study to investigate how well stimuli-based gaze analytics can be utilized to enhance motivation and learning in Massive Open Online Courses (MOOCs). Our work seeks to provide insights on how gaze variables can provide students with gazeaware feedback and help us improve the design, interfaces and analytics used as well as provide a first step towards gaze-aware design of MOOCs to amplify learning.

(c) The Author(s). 2020 Open Access This article is licensed under a Creative Commons Attribution 4.0 International License, which permits use, sharing, adaptation, distribution and reproduction in any medium or format, as long as you give appropriate credit to the original author(s) and the source, provide a link to the Creative Commons licence, and indicate if changes were made. The images or other third party material in this article are included in the article's Creative Commons licence, unless indicated otherwise in a credit line to the material. If material is not included in the article's Creative Commons licence and your intended use is not permitted by statutory regulation or exceeds the permitted use, you will need to obtain permission directly from the copyright holder. To view a copy of this licence, visit http://creativecommons.org/licenses/by/4.0/. 
The evidence for understanding and supporting users' learning is still very limited, considering the wide range of data produced when the learner interacts with a system (e.g., gaze Prieto, Sharma, Dillenbourg, \& Jesús, 2016). Devices like eye-trackers have become readily available and have the capacity to provide researchers with unprecedented access to users' attention Sharma, Jermann, \& Dillenbourg, 2014). Thus, besides commonly used variables coming from users' click-streams, keywords and preferences, we can also use eye-tracking variables to accurately measure students' attention during their interaction with learning materials (e.g., MOOC lectures).

A multitude of factors affect academic performance of the students: previous grades (Astin, 1971), students' efforts and motivation (Grabe \& Latta, 1981), socioeconomic differences (Kaplan, 1982), quality of schooling (Wiley, 1976), attention (Good \& Beckerman, 1978) and participation (Finn, 1989). In this contribution, we address the general question of how gaze-variables (related to students' reading and attention) can help students to watch MOOC videos more efficiently? We tackle this question from a teacher's perspective (how much student follows the teacher) and call it this gaze-based measure "with-me-ness". With-me-ness is defined in two levels: (1) perceptual (following teacher's deictic acts) and (2) conceptual (following teacher discourse). Specifically, in this contribution, we address the following two questions:

\section{How eye-tracking behaviour mediates the relationship between students' mo- tivation and learning within a MOOC?. \\ 2. How well we can predict the learning gain and motivation from the eye- tracking data in its most basic form?}

In order to answer these questions, we define variables using the stimulus (video lecture) presented to the students. These variables are defined using information from the stimulus with the different levels of details. Once, we have the variables, we perform mediation analysis to answer the first questions. To answer the second question, we utilize one of the most basic eye-tracking visualisations, "Heat-maps" (Špakov \& Miniotas, 2007) to extract features and use state-of-the-art machine learning algorithms to predict the students' learning gains.

\section{Related work}

\section{Video based learning}

The use of educational videos has been widely employed in the past years. Educational videos is a vital element in several online learning forms (in a MOOC, or how-to video tutorial), students spend enormous amount of time watching various forms of educational videos (Seaton, Bergner, Chuang, Mitros, \& Pritchard, 2014). Educational videos have been studied extensively during the last decades, through the lenses of empirical studies and theories (Giannakos, 2013). One of the most commonly acceptes theoretical angles it the one of the Cognitive Theory of Multimedia Learning (CTML, Mayer \& Moreno, 2003), CTML provides several insights on how video-based learning (and multimedia in general) can be used effectively.

Paivio (2013) argued that information provided by both auditory and visual channels should increase recall and retention. Studies by Mayer and Moreno (2003) have shown 
that visual information helps to process and remember verbal information and vice versa. This argument was strengthened by cue-summation theory showing that learning performance in the combined audio and pictures was better than in the combined audio and text, if the numbers of available cues or stimuli are increased (Severin, 1967). The major benefits of video as a facilitator of educational content include presentation of detailed information (with text and image), efficient engagement of students' attention, simulating discussions and providing concrete real life examples with visualizations (Schwartz \& Hartman, 2007).

During the last year, video-based learning practices are applied in a variety of ways, such as the flipped classroom, small private online courses (SPOCs), and xMOOCs. Today, advanced video repository systems have seen enormous growth (e.g. Khan Academy, PBS Teachers, Moma's Modern Teachers, Lynda) through social software tools and the possibilities to enhance videos on them (Giannakos, 2013).

Existing research on video-based learning involves many features of today's MOOCs lectures. Volery and Lord (2000) identified 3 success factors in online education: usable and interactive technology design, instructors' enthusiasm and interest in the tool and students' exposure to the web. Tobagi (1995) developed an online distant learning system to capture lectures real time, compress them, store them on an on-demand system and transmit the videos to internal server. The on-demand video system server eliminated distance limitations and provided time independent access to study material.

Tobagi (1995) compared different modalities of video lectures (interactive video, instructional television and television) and preconceptions of difficulty for different modalities and found that there was no significant difference in the learning outcome but there was a significant difference in the level of preconceived difficulty in television and interactive videos. Cennamo, Savenye, and Smith (1991) studied the effect of video based instruction on students problem solving skills and attitude towards mathematics and instruction and concluded that there was a significant improvement after the treatment in students problem solving and mathematical skills as well as the instructional attitude.

Choi and Johnson (2005) compared learning outcome and learners motivation (attention, relevance, confidence, satisfaction) in video based learning to traditional textualinstruction based learning and found no difference in learning outcome for the two conditions. However, the students wore more attentive in video based learning condition that the textual-instruction condition.

Video lectures have several affordances besides those relying to traditional fast-forward and rewind interactions. Innovative features, such as slide-video separation, social categorization and navigation, and advanced search, have also been used recently in video learning platforms (Giannakos, Chorianopoulos, \& Chrisochoides, 2015). All this amount of interactions can be converted via analytics into useful information that can be used to support learning (Kim et al., 2014). As the number of learners and the diversity of collected data grows, our ability to capture richer and more authentic learning patterns grows as well, allowing us to create new affordances that amplify our learning capacity.

\section{Eye-tracking and education}

Utilizing representative and accurate data allows us to better understand students and design meaningful experiences for them. Eye tracking has been employed to understand 
the learning processes and different levels of outcome in a multitude of learning scenarios. Prieto et al. (2016), used eye-tracking data to explain the cognitive load that the teachers experience during different classes and various scenarios. These scenarios include different factors such as experience of the teacher, size of the class, presence of a new technology and presence of a teaching assistant. The results show that the eyetracking data is an important source of information explaining different factors in teachers' orchestration load and experience.

Eye-tracking has also been used in online learning for both in individual (Kizilcec, Papadopoulos, \& Sritanyaratana, 2014) and collaborative (Sharma, Caballero, Verma, Jermann, \& Dillenbourg, 2015a, b) settings. Sharma et al. (2014) focus on capturing the attention of the individual learners in a video-based instructional setting to find the underlying mechanisms for positive learning outcome; Sharma et al. (2015a, b) also focus on joint attention in remote collaborative learning scenarios to predict the learning outcome.

In general, eye-tracking allows us to generate rich information, but it can be challenging to identify what information is processed and retained based on human's gaze. The eye-mind hypothesis (Just \& Carpenter, 1980) proposes that there is a connection between people gaze and attention, if people process the information that they visually attend to. In this contribution, we utilize eye-tracking to measure students' attention and then address how students' attention (i.e., "with-me-ne") has the capacity to mediate the relationship between students' motivation and learning within a MOOC video.

\section{Methodology}

\section{Participants and procedure}

A total of 40 university students (12 females) from a major European university participated in the experiment. The only criterion for selecting the participant was that each participant took the introductory Java course in the previous semester. This is also a prerequisite for taking the Functional Programming in Scala course at the university campus. The participants watched two MOOC videos from the course "Functional Programming Principles in Scala" and answered programming questions after each video.

Upon their arrival in the experiment site the participants signed a consent form and answered the study processes questionnaire (SPQ, Biggs, Kember, \& Leung, 2001). Then watched the two MOOC videos and answered the quiz based on what they were taught in the videos. During their interaction with the MOOC videos their gaze was recorded, using SMI RED 250 eye-trackers.

Some of the reasons why 40 students are sufficient in our study are: (i) the data collected are "big" in terms of the $4 \mathrm{Vs}$ ' (volume, variety, veracity, velocity). For example, eyetracking data collected at a high frequency (e.g., $250 \mathrm{~Hz}$ in the present study) means that we have a continuous and unobtrusive measurement of the behaviour of the users. Collecting this kind of data results into continuously and massively gathering a few Gigabytes of data per person (Volume and Velocity). Furthermore, collecting data in the form of multiple datatypes at once (i.e., fixations, saccades, heatmaps, scanpaths, clickstream) satisfies Variety, whereas, previous research has acknowledged those data for cognitive load, attention, anticipation, fatigue, information process (Veracity); (ii) the current cost of the equipment necessary to collect those data does not allow for simultaneous use of multiple eye-trackers, but the granularity of information we can have access to, justifies their usage. 
Based on these reasons it is safe to say that 40 participants are indeed enough to arrive at the conclusions that our paper is deriving with the present study.

Moreover, in recent eye-tracking research we see similar sizes of the population used. For example, in two recent systematic reviews (Alemdag \& Cagiltay, 2018; Ashraf et al., 2018) with a combined 85 different eye-tracking studies the majority (84.71\%) of the studies had between 8 and 60 participants. The papers cited in this contribution with eye-tracking research also have the number of participants between 10 and 40 participants (except the collaborative studies where the researchers had 28 to 40 pairs).

\section{Measures}

The measures used in our study were: content coverage, scanpath (a combination of the fixations and saccades in the order of appearances) based variables, students/ teacher co-attention (i.e., with-me-ness) coming from eye-tracking, students' motivation coming from SPQ and students learning (coming from the final test).

The eye-tracking variables are defined using the semantics of the stimulus, that is the video lecture in our case. We define eye-tracking variables at four levels (see Table 1). First, the content coverage has no semantics from the video. Second, the scanpath based variables required us to define areas of interest on the video. Third, the perceptual with-me-ness was computed using the areas of interest and the pointing gestures of the teacher. Finally, the conceptual with-me-ness was defined using the areas of interest definitions and the dialogue of the teacher.

\section{Content coverage}

Content coverage is computed using the heat-maps (for details on heat-maps see Holmqvist et al. 2011) of the participants. We divided the MOOC lecture in slices of $10 \mathrm{~s}$ each and computed the heat-maps for each participant. Following are the steps to compute attention points from the heat-maps:

1. Subtract the image without heat-map (Fig. 1b) from the image that has the heatmap (Fig. 1a).

2. Apply connected components on the resulting image (Fig. 1c)

3. The resulting image with connected components identified (Fig. 1d) gives the attention points.

4. The combined area of attention points in a given time window represents the content coverage of that time window.

Attention points typically represented the different areas where the students focused their attention. The number of the attention points would depict the number of attention zones and the area of the attention points (Content Coverage) would depict the total time spent on a particular zone. We used the area covered by attention points per $10 \mathrm{~s}$ to check for the mediation effect on the relationship across the levels of performance and learning motivation. The area covered by the attention points typically indicated the content coverage for students. The content coverage indicates the content read by the students and the time spent on the content. 


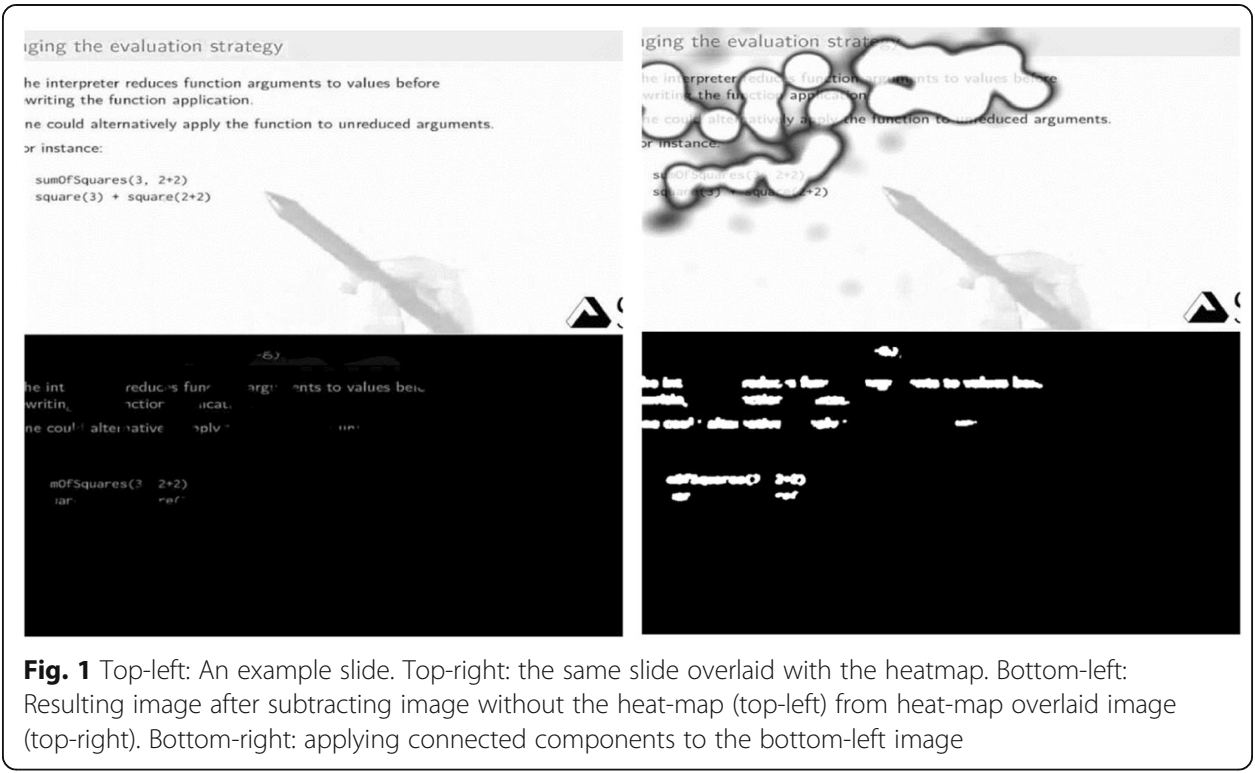

\section{Scanpath based variables}

AOI misses An area of interest (AOI) was said to be missed by a participant who did not look at that particular AOI at all during the period the AOI was present on the screen. In terms of learning behaviour AOI misses would translate to completely ignoring some parts of the slides. We counted the number of such AOIs per slide in the MOOC video as a scan-path variable and compare the number of misses per slide across the levels of performance and learning strategy (for details on areas of interest see Holmqvist et al. 2011).

AOI backtracks A back-track was defined as a saccade that went to the AOI which is not in the usual forward reading direction and had already been visited by the student. For example, in the Fig. 2, if a saccade goes from AOI3 to AOI2 it would be counted as a back-track. AOI back-tracks would represent rereading behaviour while learning from the MOOC video. The notion of term rereading in the present study was slightly different than what is used in existing research (for example, Millis and King (2001), Dowhower (1987) and Paris and Jacobs (1984)). The difference comes from the fact that in the present study the students did not reread the slides completely but they can refer to the previously seen content on the slide until the slide was visible. We counted the number of back-tracks per slide in the MOOC video as a scan-path variable and

Table 1 Eye-tracking measurements and level and type of semantics involved in defining those variables

\begin{tabular}{lll}
\hline Eye-tracking Measurements & Level of semantics & Type of semantics involved \\
\hline Content Coverage & Low & Only Stimulus \\
Scanpath based variables & Medium & Areas of interest definition \\
Perceptual with-me-ness & High & Areas of interest definition and Teachers' gestures \\
Conceptual with-me-ness & Highest & Areas of interest definition and Teachers' dialogues \\
\hline
\end{tabular}




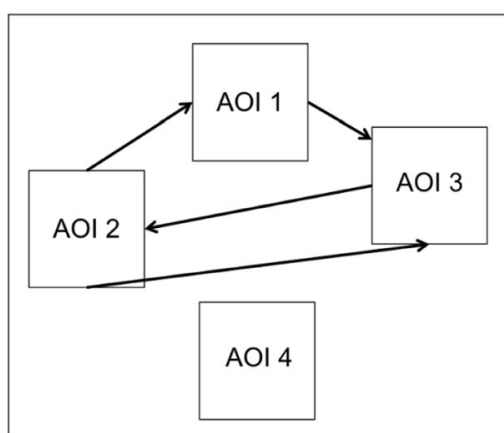

Scan-path: $\mathrm{AOI} 1$-> AOI3 -> AOI2 -> $\mathrm{AOI} 3$

Number of $A O I$ backtracks: 1 (AOI3)

Number of AOI misses: 1 (AOl4)

Fig. 2 A typical example of a scanpath (left); and the computation of different variables (right)

compared the number of back-tracks per slide across the levels of performance and motivation (Fig. 3 shows the typical AOIs on a slide).

\section{With-me-ness}

With-me-ness measures how much the student is paying attention to what the teacher is saying or pointing at (Sharma et al., 2014; Sharma et al., 2015a, b). With-me-ness is defined at two levels of teacher-student interaction: perceptual and conceptual.

\section{Perceptual with-me-ness}

Perceptual with-me-ness measures if the student looks at the items referred to by the teacher through deictic acts (sometimes accompanied by words like, here, this variable or only by verbal references, like, the counter, the sum). Deictic references are implemented by using two cameras during MOOC recording, one that captures the teacher's face and one, above the writing surface, that captures the hand movements. In some MOOCs, the hand is not visible but teacher uses a digital pen whose traces on the display (underlining a word, circling an object, adding an arrow) act as a deictic gestures.

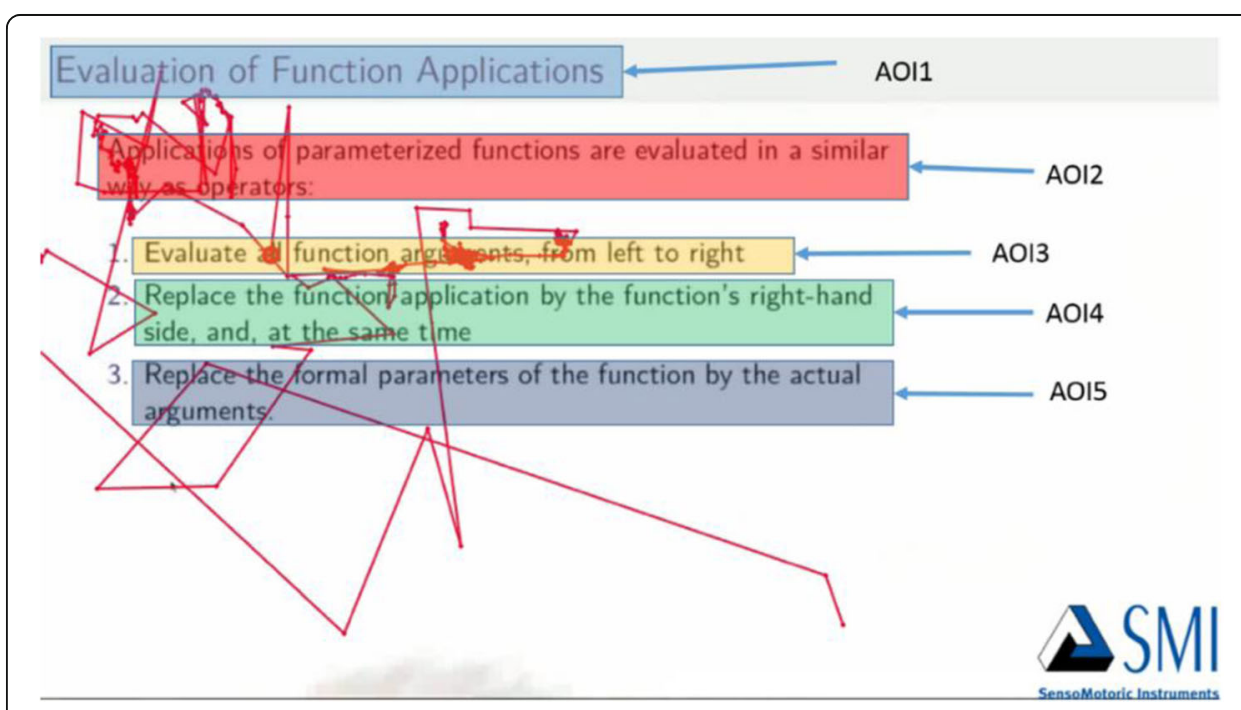

Fig. 3 Example of a scan-path and Areas of Interest (AOI) definition. The rectangles show the AOls defined for the displayed slide in the MOOC video and the red curve shows the visual path for $2.5 \mathrm{~s}$ 
The perceptual "With-me-ness" has 3 main components: entry time, first fixation duration and the number of revisits (Fig. 4). Entry time (Fig. 4 top-right) is the temporal lag between the times a referring pointer appears on the screen and stops at the referred site $(x, y)$ and the first time the student's gaze stops at $(x, y)$. First fixation duration (Fig. 4 bottom-left) is how long the student gaze stops at the referred site for the first time. Revisits (Fig. 4 bottom-right) are the number of times the student gaze comes back to the referred site. The measure of perceptual with-me-ness is an arithmetic combination of these components $($ FFD $=$ First Fixation Duration; ET = Entry Time; NumRV = Number of revisits; $\mathbf{R V}=$ Re Visit duration):

$$
\text { Perceptual With_me_ness }=\frac{\text { FFD }-E T+\sum_{i=1}^{N u m R V} R V_{i}}{\text { Total duration of the deictic reference }}
$$

The with-me-ness measurement has also been used by Sharma et al. (2014 and $2015 \mathrm{a}, \mathrm{b})$ to measure how much time the students spent in following the teacher's deictics and dialogues. Sharma et al. (2014 and 2015a, b) found this measure to be correlated to the learning gains of the students. We have extended the analyses to include the student motivation as an independent variable, learning as the dependent variable and gaze behavior as the mediating variable.

\section{Conceptual with-me-ness}

Conceptual with-me-ness is defined by the discourse of the teacher (i.e., to what extend students look at the object that the teacher is verbally referring to) Fig. 5 provides an example. Thus, conceptual with-me-ness measures how often a student looks at the

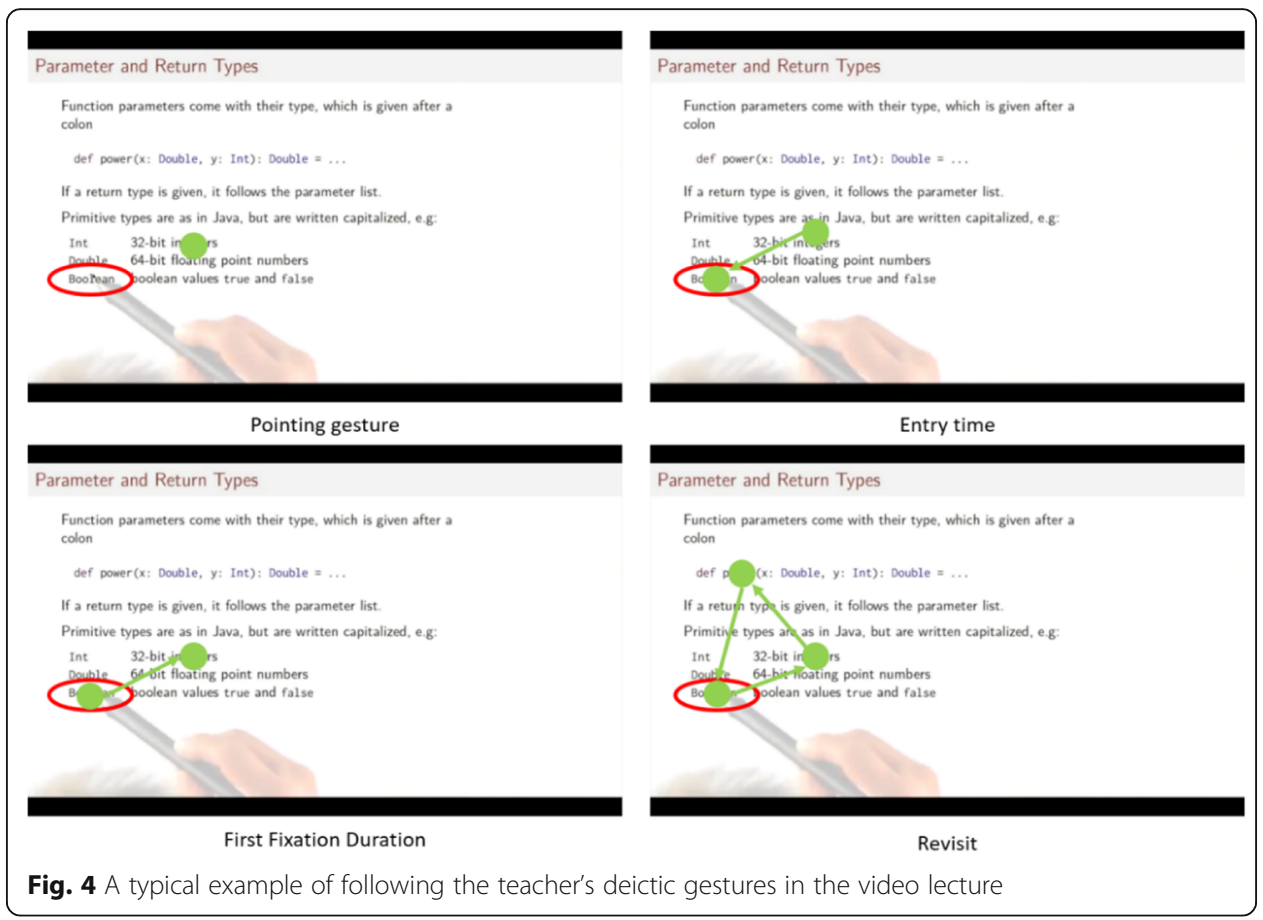




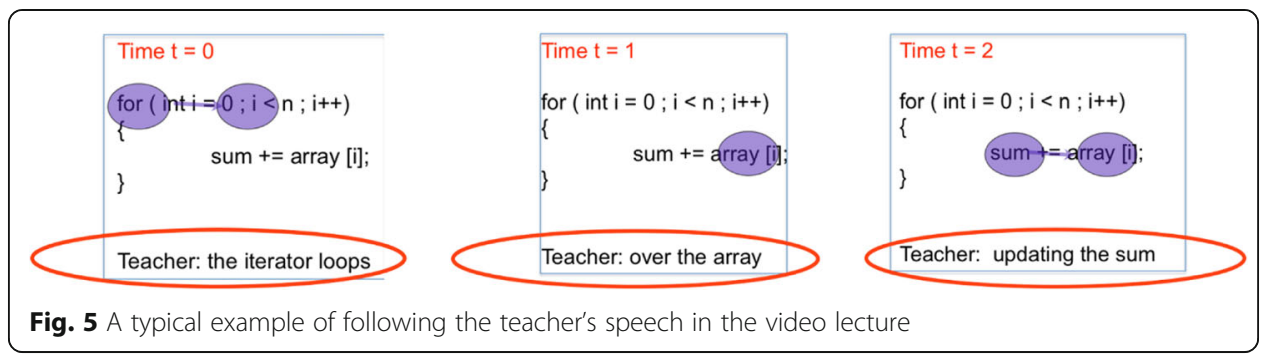

objects verbally referred to by the teacher during the whole course of time (the complete video). In order to have a consistent measure of conceptual "With-me-ness" we normalize the time a student looks at the overlapping content by slide duration.

\section{Motivation}

We used the motivation scales from the SPQ (Biggs et al., 2001). This is a 5-point Likert scale questionnaire containing 10 questions ( 5 for deep and 5 for surface motivation). Deep motivation is defined as having the intrinsic motivation towards learning, while the surface motivation is defined as fear of failing in the tests ((Biggs et al., 2001)). In this study we used the mean motivation (mean of deep and surface) that has an average value of 2.10 (Std. Dev. $=1.20)$.

\section{Learning}

At the end of the videos the students took a test about the content they were taught in the two videos. The score from this test was considered to be the learning performance in this paper. After this point, we would refer to this as learning. The mean learning value was 6.9 out of 10 (Std. Dev. = 1.6). For the test, the instructor of the MOOC helped the authors to create the multiple-choice quiz for the two videos. This quiz was similar to the one used in the MOOC running at Coursera platform.

\section{Data analysis}

\section{Mediation analysis}

To identify how "with-me-ness" (measured by eye-tracking) mediates the relationship between students' motivation (measured by the questionnaire) and learning (measured by the post quiz) within a MOOC we employ mediation analysis proposed by Baron and Kenny (1986). In our analysis, we used motivation as the predictor, learning as the outcome and gaze behaviour as the mediating variables. Figure 6 shows the schematic representation of the model.

To examine with-me-ness capacity to mediate the relationship between motivation and learning we followed Baron and Kenny (1986) three steps process: a) the predictor (i.e., motivation) must significantly influence the mediator (i.e., with-me-ness); b) the predictor (i.e., motivation) must significantly influence the outcome (i.e., learning); c) both predictor and mediator are employed to predict the outcome: if both of them significantly affect the dependent variable, then this mediator partially mediates the impact of the predictor independent variable on the outcome; if the influence of mediator is significant but the influence of predictor is not, then mediator fully mediates the impact of predictor on outcome. 


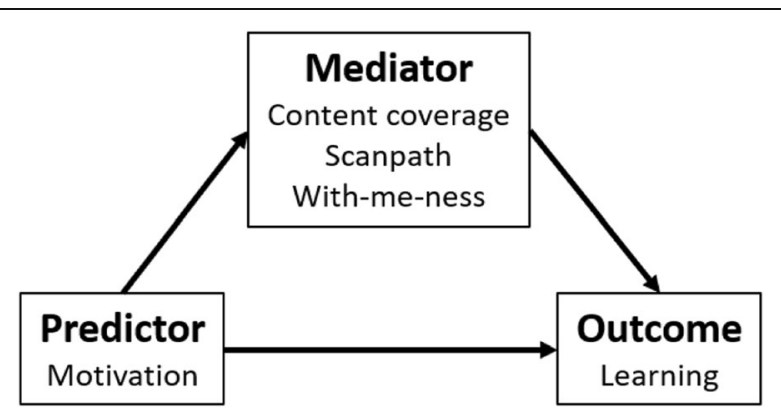

Fig. 6 Schematic representation of mediation effect and the example from the present contribution

Learning outcome and motivation prediction: feature extraction

For predicting the learning outcome from the behaviour data, we used the heat-maps and a pretrained deep neural network to generate the features. Figure 7 shows the basic working pipeline to extract the features from the heatmap image to the basic feature vector. Following are the steps to extract features from the eye-tracking data and the video lecture.

1. Overlay the eye-tracking data on the video to create the heatmap.

2. Create the heatmap image from every presentation slide in the video lecture (this step resulted into 15 heatmap image per participant).

3. Use the pretrained VGG-19 (Simonyan \& Zisserman, 2014) deep neural network architecture to extract the 1000 features per image.

4. Use a non-overlapping and sliding window of size 10 to reduce the number of features to 100 .

Feature selection for learning outcome and motivation prediction: least absolute shrinkage selection operator

To select the most important features we employ the Least Absolute Shrinkage and Selection Operator (LASSO, Tibshirani, 1996). LASSO is an extension of Ordinary Least Square (OLS) regression techniques fit for the cases where the number of examples is

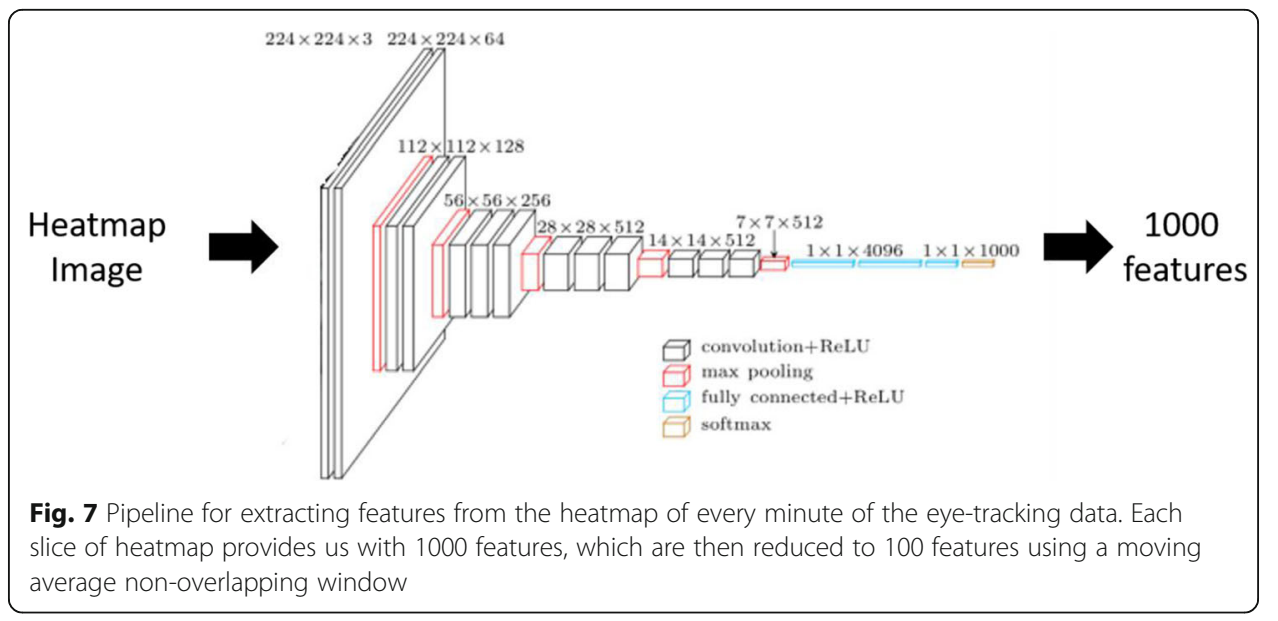


less than the length of the feature vector (Tibshirani, 1996). To find the best fitting curve for a set of data points, OLS tries to minimize the Residual Sum of Squares (RSS) which is the difference between the actual values of the dependent variable $y$ and the fitted values $\hat{y}$. The formulation of the OLS is given as follows:

$$
\hat{y}=\alpha_{0}+\beta_{1} x_{1}+\beta_{2} x_{2}+\ldots+\beta_{n} x_{n}
$$

The objective of the OLS regression is to minimize the difference between $\sum(\hat{\mathrm{y}}-y)^{2}$ with the constraint that $\sum \beta_{i}^{2} \leq s$. Where $s$ is called the shrinkage factor. LASSO on the other hand performs similar optimization with the slight difference in the constraint, which is now $\Sigma\left|\beta_{i}\right| \leq s$. While using LASSO, some of the $\beta_{i}$ will be zero. Choosing $s$ is like choosing the number of predictors in a regression model. Cross-validation can be used to estimate the best suited value for $s$. Here, we used a 10-fold cross-validation to select the appropriate value of $s$.

\section{Learning outcome and motivation prediction and prediction evaluation}

In order to predict the learning outcome of the students, we used several prediction algorithms. These algorithms include Gaussian process models (Rasmussen, 2003) with linear and polynomial kernels, Support Vector machines (SVM, Scholkopf \& Smola, 2001) also with linear and polynomial kernels, Random forest (Liaw \& Wiener, 2002), Generalised Additive Models (GAM, Hastie (1993) and Hastie and Tibshirani, 1990). The main reason for using these particular algorithms is that these are designed to handle datasets that have high frequency for fewer examples.

We divided the whole dataset into training ( $80 \%, 32$ students) and testing $(20 \%, 8$ students). For removing the selection bias from the training set, we performed a 5-fold cross-validation. The results reported are the average error rate for all the crossvalidation iterations.

For evaluating our prediction results, we are using the Normalized Root Mean Squared Error (NRMSE). NRMSE is the proposed metric for student models (Pelánek, 2015), and is used in most of the articles in learning technology (Moreno-Marcos, Alario-Hoyos, Muñoz-Merino, \& Kloos, 2018) for measuring the accuracy of learning prediction.

\section{Results}

To answer the first research question about the mediation effect of the gaze behaviour on the relation between learning and motivation, we will present the mediation analyses with content coverage, scanpath variables and with-me-ness. Further, to answer the second research question about the predicting ability of simplistic gaze variables, we will present the prediction results for both the students' motivation and their learning.

\section{Content coverage}

To examine the mediation effect of content coverage we tested the model shown in Fig. 8. As shown in Table 2, the direct link between motivation and both variables of content coverage was significant and hence satisfied the first condition. The link between motivation and learning was also significant and hence satisfied the second condition as well. Moreover, the direct relationship between motivation with learning was 


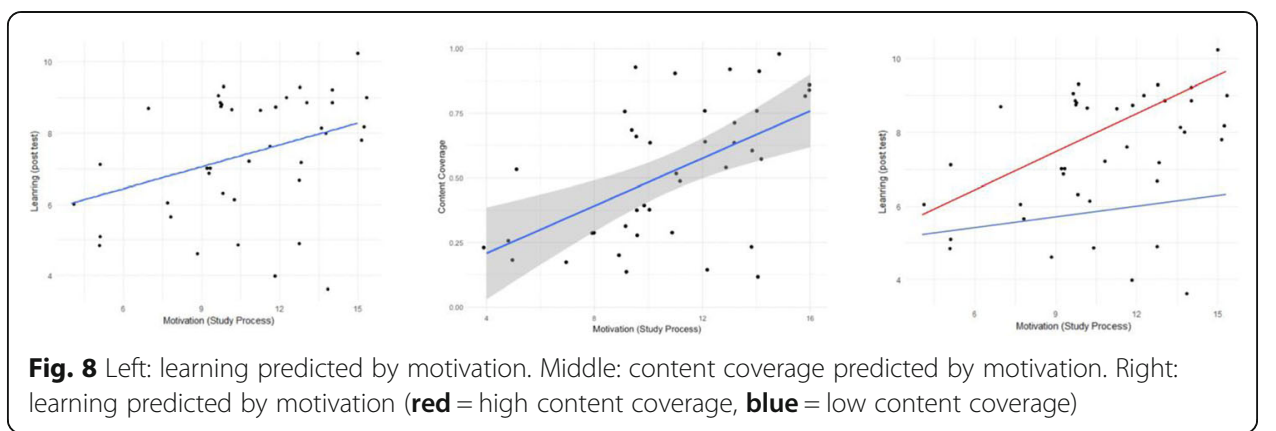

not significant when content coverage variable were added. In Table 2 we present the results of the mediation analyses (row one for content coverage).

We observe that learning can be significantly predicted by motivation and that content coverage can be predicted by motivation. Finally, there is a significant prediction of learning by motivation and content coverage, however the coefficient of motivation is not significant anymore. Thus we can conclude that the content coverage fully mediates the relation between motivation and learning. The positive correlation between the motivation and learning is higher for the students with the higher content coverage than the positive correlation between motivation and learning for the students with the lower content coverage. It is clear from Fig. 8 that the students with high motivation have higher chances of getting a high score if they have high content coverage than the students with lower motivation.

\section{Scanpath variables}

To examine the mediation effect of scanpath variables we tested the model shown in Fig. 9 with both the AOI misses and the AOI backtracks of scanpath variables. As shown in Table 2, the direct link between motivation and both scanpath variables was significant and hence satisfied the first condition. The link between motivation and learning was also significant and hence satisfied the second condition as well. However, the direct relationship between motivation with learning was still significant when scanpath variables (misses and backtracks) were added. In Table 2 we present the results of the two mediation analyses (row two for AOI misses and row three for AOI backtracks).

We observe that learning can be significantly predicted by motivation and that perceptual with-me-ness can be predicted by motivation. Finally, there is a significant prediction of learning by motivation and AOI backtracks, however the coefficient of motivation is

Table 2 Mediating effect tests

\begin{tabular}{|c|c|c|c|c|c|c|c|}
\hline \multirow{2}{*}{$\begin{array}{l}\text { Predictor } \\
(\mathrm{Pr})\end{array}$} & \multirow[t]{2}{*}{ Mediator (M) } & \multirow{2}{*}{$\begin{array}{l}\text { Outcome } \\
\text { (O) }\end{array}$} & \multirow{2}{*}{$\begin{array}{l}\mathrm{Pr} \\
\mathrm{M}\end{array} \rightarrow$} & \multirow{2}{*}{$\begin{array}{l}\mathrm{Pr} \rightarrow \\
\mathrm{O}\end{array}$} & \multicolumn{2}{|c|}{$\mathrm{Pr}+\mathrm{M} \rightarrow \mathrm{O}$} & \multirow[t]{2}{*}{ Mediating effect } \\
\hline & & & & & $\overline{\mathrm{Pr}}$ & $M$ & \\
\hline Motivation & Content coverage & Learning & $3.98^{* * *}$ & $2.40 *$ & 1.35 & $3.09^{* * *}$ & Full mediation \\
\hline Motivation & $\mathrm{AOI}$ misses & Learning & $-2.26^{* *}$ & $2.40 *$ & $2.04^{*}$ & $-2.39^{*}$ & Partial mediation \\
\hline Motivation & AOI backtracks & Learning & $2.41^{*}$ & $2.40 *$ & $2.15^{*}$ & $2.58^{*}$ & Partial mediation \\
\hline Motivation & Perceptual with-me-ness & Learning & $2.69^{* *}$ & $2.40^{*}$ & 1.40 & $2.30^{* *}$ & Full mediation \\
\hline Motivation & Conceptual with-me-ness & Learning & $2.05^{* *}$ & $2.40^{*}$ & 1.57 & $2.90^{* *}$ & Full mediation \\
\hline
\end{tabular}

$\left({ }^{*} p<.05 ;{ }^{* *} p<.01 ;{ }^{* * *} p<.005\right)$ 


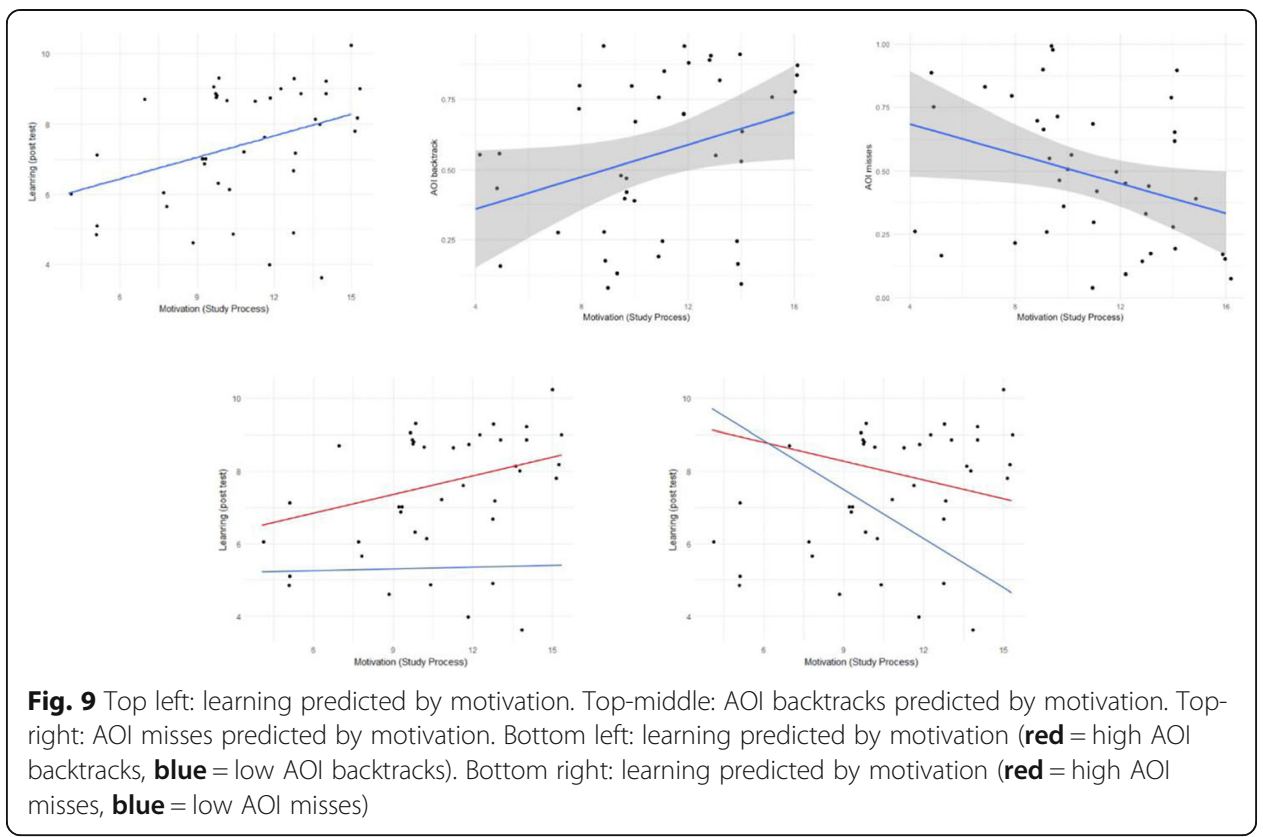

still significant. Thus we can conclude that the AOI backtracks only partially mediates the relation between motivation and learning. We can see that the correlation between the motivation and the learning is more positive for the students with high number AOI backtracks than that for the students with low number of AOI backtracks. It is clear from Fig. 9 that the students with high motivation have higher chances of getting a high score if they perform more AOI backtracks than the students with lower motivation.

Next, we observe that that learning can be significantly predicted by motivation, and that AOI misses can be predicted by motivation. Also, there is a significant prediction of learning by motivation and AOI misses, however the coefficient of motivation is still significant anymore. Thus we can conclude that the AOI misses only partially mediates the relation between motivation and learning. We can see that the correlation between the motivation and the learning is more negative for the students with high number AOI misses than that for the students with low number of AOI misses. It is clear from Fig. 9 that the students with low motivation have higher chances of getting a low score if they miss more AOIs than the students with higher motivation.

\section{With-me-ness}

To examine the mediation effect of with-me-ness we tested the model shown in Fig. 10 with both the perceptual and the conceptual variables of with-me-ness. As shown in Table 2 , the direct link between motivation and both variables of with-me-ness was significant and hence satisfied the first condition. The link between motivation and learning was also significant and hence satisfied the second condition as well. Moreover, the direct relationship between motivation with learning was not significant when with-me-ness variables (perceptual and the conceptual) were added. In Table 2 we present the results of the two mediation analyses (row four for perceptual and row five for conceptual with-me-ness).

We observe that learning can be significantly predicted by motivation and that perceptual with-me-ness can be predicted by motivation. Finally, there is a significant 


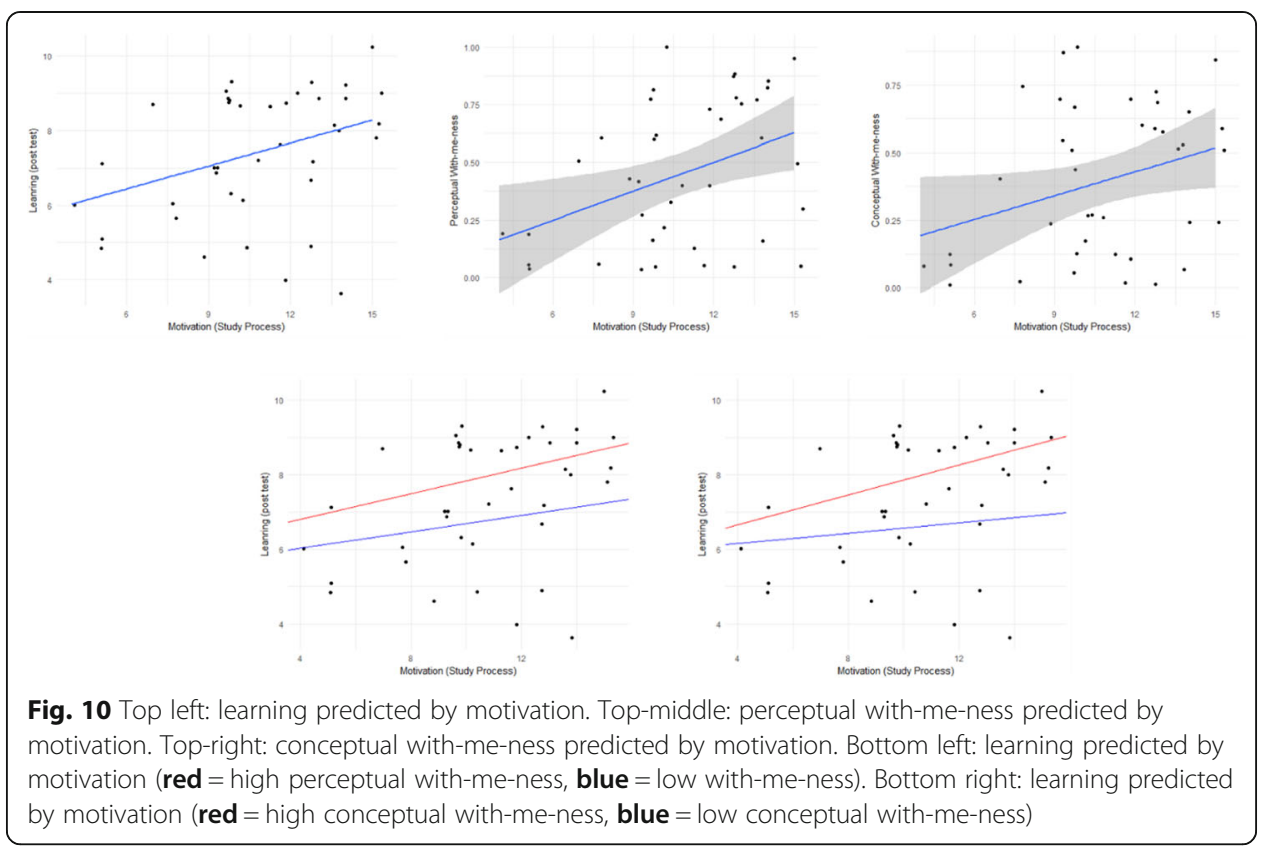

prediction of learning by motivation and perceptual with-me-ness, however the coefficient of motivation is not significant anymore. Thus, we can conclude that the perceptual with-me-ness fully mediates the relation between motivation and learning. We can see that the correlation between the motivation and the learning is more positive for the students with high perceptual with-me-ness than that for the students with low perceptual with-me-ness. It is clear from Fig. 10 that the students with high motivation have higher chances of getting a high score if they high perceptual with-me-ness than the students with lower motivation.

Next, we observe that that learning can be significantly predicted by motivation, and that conceptual with-me-ness can be predicted by motivation. Also, there is a significant prediction of learning by motivation and conceptual with-me-ness, however the coefficient of motivation is not significant anymore. Thus we can conclude that the conceptual with-me-ness fully mediates the relation between motivation and learning. We can see that the correlation between the motivation and the learning is more positive for the students with high conceptual with-me-ness than that for the students with low conceptual with-me-ness. It is clear from Fig. 10 that the students with high motivation have higher chances of getting a high score if they high conceptual with-me-ness than the students with lower motivation.

\section{Prediction results}

Figure 11 shows the prediction results for the students' learning and motivation. For learning prediction, we observed a minimum error of $5.04 \%(\mathrm{SD}=0.52 \%)$ using the Gaussian Process Model with a polynomial kernel. The second least error of $8.07 \%$ $(\mathrm{SD}=0.54 \%)$ was obtained using a Support Vector Machine also with a polynomial kernel. The worst error rate was found to be $11.18 \%$ ( $\mathrm{Sd}=0.63 \%$ ) using the Generalised additive models. For motivation prediction, we observed similar performances with the prediction algorithms. We observed a minimum error of $9.04 \%(\mathrm{SD}=0.56 \%)$ using the 


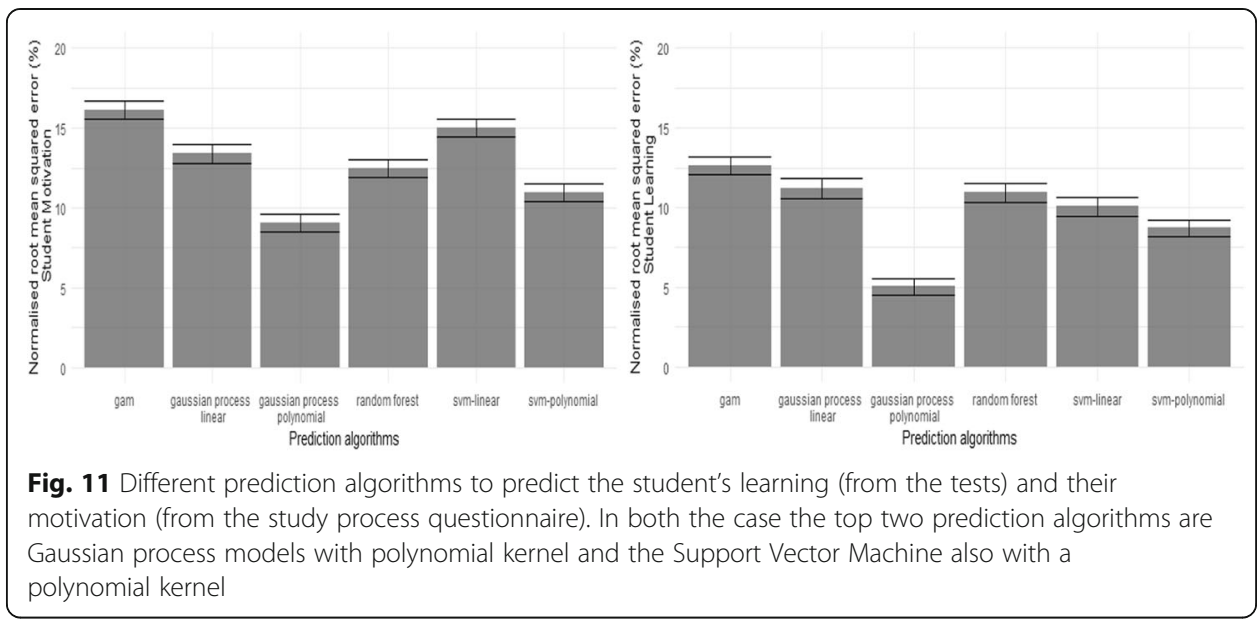

Gaussian Process Model with a polynomial kernel. The second least error of $10.98 \%$ $(\mathrm{SD}=0.57 \%)$ was obtained using a Support Vector Machine also with a polynomial kernel. The worst error rate was found to be $16.11 \%(\mathrm{Sd}=0.63 \%)$ using the Generalised additive models.

\section{Discussions and conclusions}

The reported study developed and empirically explored two models, where teacher/student co-attention (i.e., with-me-ness) were found to mediate the relationship of motivation and learning in MOOC videos. These two models demonstrated how the aspect of co-attention, not only influences learning, but also affects the effect of motivation in learning. Quantifying an often-overlooked element (i.e., instructor's capacity to draw student's attention) in online courses.

The attention points, derived from the heat-maps, were indicative of the students' attention both in the terms of screen space and time. The area of the attention points depended on the time spent on a specific area on the screen. Higher average area of the attention points could be interpreted as more reading time during a particular period. The good performing students having a higher motivation had the highest content coverage (larger areas of the attention) among all the participants, despite having spent the similar time on the slides.

However, more reading time did not always guarantee higher performance. Byrne, Freebody, and Gates (1992) showed the inverse in a longitudinal reading study by proving that the best performing students were the fastest readers. On the other hand, Reinking (1988) showed that there was no relation between the comprehension and reading time. As Just and Carpenter (1980) put "There is no single mode of reading. Reading varies as a function of who is reading, what they are reading, and why they are reading it." The uncertainty of results about the relation between the performance and the reading time led us to find the relation between the reading time, performance and learning motivation. We found that the good-performers had more reading time than poor-performers and the high motivated-learners had more reading time than low motivated-learners. We could interpret this reading behaviour, based upon the reading time differences, in terms of more attention being paid by the good performing students having a high learning motivation than other student profiles. We could use 
content coverage to give feedback to the students about their attention span. Moreover, one could use the content coverage for student profiling as well based on the performance and the learning motivation.

The area of interest (AOI) misses and back-tracks were the temporal features computed from the temporal order of AOIs looked at. We found that good-performers with high motivation had significantly fewer AOI misses than the poor-performers with low motivation. AOI misses could be useful in providing students with the feedback about their viewing behaviour just by looking at what AOIs they missed.

The AOI back-tracks were indicative of the rereading behaviour of the students. We found that the good performers and highly motivated learners had significantly more backtracks than the poor-performers. Moreover, some of the good-performers back-tracked to all the previously seen content, this explains the special distribution of AOI back-tracks for good-performers. Millis and King (2001) and Dowhower (1987) showed in their studies that rereading improved the comprehension. In the present study, the scenario is somewhat different than Millis and King (2001) and Dowhower (1987). In the present study, the students did not read the study material again. Instead, the students referred back to the previously seen content again during the time the slide was visible to them. Thus, the relation between rereading of the same content and the performance should be taken cautiously, clearly further experimentation is needed to reach a causal conclusion.

One interesting finding in the present study was the fact that the content coverage had fully mediated the relation between the performance and the learning motivation. Whereas, the AOI misses and AOI back-tracks had partial mediation effects. This could be interpreted in terms of the type of information we considered to compute the respective variables. For example, the content coverage computation took into account both the screen space and the time information and AOI back-tracks (and misses) computation required only the temporal information. However, in the context of the present study, we could not conclude the separation between spatial and temporal information and how it effected the relation between the gaze variables and performance and learning strategy.

In addition, we found that high-performers (those who scored high in the test) had more perceptual with-me-ness on the referred sites than the low-performers. This is in accordance with the literature, where Jermann and Nüssli (2012), showed that better performing pairs had more recurrent gaze patterns during the moments of deictic references. We also found that the students who scored better in the test, were following the teacher, both in deictic and discourse, in an efficient manner than those who did not score well in the test. These results were not surprising, but could be utilised to inform the students about their attention levels during MOOC lectures in an automatic and objective manner. The results also contribute towards our long-term goal of defining the student profiles based on their performance and motivation using the gaze data. The attention points can serve the purpose of a delayed feedback to the students based on their attention span.

The conceptual with-me-ness can be explained as a gaze-measure for the efforts of the student to sustain common ground within the teacher-student dyad. Dillenbourg and Traum (2006) and Richardson, Dale, and Kirkham (2007) emphasised upon the importance of grounding gestures to sustain shared understanding in collaborative problem solving scenarios. A video is not a dialogue; the learner has to build common grounds, asymmetrically, with the teacher. The correlation we observed between conceptual with-me-ness and the test score $(r=0.36, p<0.05)$ seemed to support this hypothesis. 
Another interesting finding of our study is that the conceptual with-me-ness has more percentage mediation than the perceptual with-me-ness (39\% for conceptual as compared to $33 \%$ for perceptual with-me-ness). This shows that eye-tracking can not only provide access to students' attention but also to the students' information processing mechanisms as well. Thus, students gaze is an important source of information that can be used to inform online learning.

Finally, from the prediction results, we were able to show that the heat-maps cannot be only used as a popular visualization tools, but also as a source of features to predict performance and other traits, such as motivation. The best prediction results for the performance was with a 5.04\% normalized error. In terms of a quiz-based evaluation of learning, which in our case are 10 questions, this error translates to less than one question. For example, if a student answers 9 questions correctly, our method will predict the score within the range of [8.5-9.5]. Similarly, on the motivation scale, which is a 5point Likert scale making it in the range of [0 -- 50], the error of $9.04 \%$ would translate to one incorrect prediction out of ten on the scale proposed by Biggs et al. (2001).

Additionally, in this contribution, the eye-tracking variables we defined had different pre-processing requirement. These variables also have capacities in terms of being used within an adaptive and real-time system. The computation of content coverage is realtime and requires no pre-processing of the data or the stimulus. The Scan-path variables can also be computed in real-time and there is small amount of pre-processing required in term of defining the area of interest (AOI) to be able to compute them. The pre-processing for computing the perceptual with-me-ness could be automatized since there are computer-vision methods to detect pointing/other deictic gestures of the teacher. Once this detection is done, the real-time computation of Perceptual with-meness if fairly straightforward. Finally, the conceptual with-me-ness, requires a few manual interventions in transcribing the teachers' dialogues and mapping them to the content. This acts as a hindrance in the real-time computation of the conceptual with-me-ness, and therefore, this is the only gaze-based measure used in this study that requires further work to be used as within a personalised adaptive gaze-based feedback system.

To gain further insight into the design of MOOC videos and the affordances of the respective systems, we need to consider eye-gaze measurements (or can call them gaze analytics) that we found to not only strongly associated with learning, but also mediate the influence of other variables (i.e., motivation). Discussing these features from a technical standpoint can give rise to practical implications for the design of MOOC videos (e.g., designed in a way to draw students' attention (Kizilcec et al., 2014) and the respective video-based learning systems (e.g., offer an indication of students' attention based on the web-camera).

For future work, we are now beginning to collect eye-tracking data from different types instruction (e.g., pair problem solving) utilizing different stimulus (e.g., not controlled from the student like the video). In addition, we intend to investigate whether a plausible association exists between different students (e.g., novices). After identifying the role of with-me-ness and other gaze-analytics in different contexts, we will be able to propose how gaze-analytics can be integrated to various contemporary learning systems. For example, allowing us to enable student profiles based on their performance and learning strategy using gaze-analytics, and ultimately provide gaze-aware feedback to improve the overall learning process. 


\section{Abbreviations}

MOOC: Massive open online course; Al: Artificial intelligence; CTML: Cognitive theory of multimedia learning; SPOC: Special purpose online courses; XMOOC: eXtended massive open online course; SPQ: Study process questionnaire; $4 \mathrm{~V}$ : Volume, variety, velocity, and veracity; AOI: Area of interest; FFD: First fixation duration; ET: Entry time; NumRV: Number of ReVisits; RV: ReVisits; Std. Dev. Or SD: Standard Deviation; VGG: Visual Geometry Group; LASSO: Least absolute shrinkage and selection operator; OLS: Ordinary least squares; RSS: Residual sum of squares; GAM: Generalised additive models; NRMSE: Normalised root mean squared error

\section{Acknowledgements}

This work is supported from the Norwegian Research Council under the projects FUTURE LEARNING (number: 255129/ H20) and Xdesign (290994/F20).

\section{Authors' contributions}

KS designed and conducted the study, analysed the data and drafted the manuscript. MG participated in the analysis of the data and framing of the contribution. PD participated in the conceptualisation and design of the study. The authors read and approved the final manuscript.

\section{Funding}

No funding was received for this study.

\section{Availability of data and materials}

As it is possible to identify participants from the data, ethical requirements do not permit us to share participant data from this study.

\section{Competing interests}

Participation was voluntarily, and all the data collected anonymously. Appropriate permissions and ethical approval for the participation requested and approved.

There is no potential conflict of interest in this study.

\section{Author details}

${ }^{1}$ Department of Computer Science, Norwegian University of Science and Technology, Trondheim, Norway.

2Department of Computer Science, École Polytechnique Fédérale de Lausanne, Lausanne, Switzerland.

Received: 4 September 2019 Accepted: 3 April 2020

\section{Published online: 26 April 2020}

\section{References}

Alemdag, E., \& Cagiltay, K. (2018). A systematic review of eye tracking research on multimedia learning. Computers in Education, 125, 413-428.

Ashraf, H., Sodergren, M. H., Merali, N., Mylonas, G., Singh, H., \& Darzi, A. (2018). Eye-tracking technology in medical education: A systematic review. Medical Teacher, 40(1), 62-69.

Astin, A. W. (1971). Predicting academic performance in college: Selectivity data for 2300 American colleges.

Baron, R. M., \& Kenny, D. A. (1986). The moderator-mediator variable distinction in social psychological research: Conceptual, strategic, and statistical considerations. Journal of Personality and Social Psychology, 51(6), 1173.

Biggs, J., Kember, D., \& Leung, D. Y. (2001). The revised two-factor study process questionnaire: R-SPQ-2F. The British Journal of Educational Psychology, 71(1), 133-149.

Byrne, B., Freebody, P., \& Gates, A. (1992). Longitudinal data on the relations of word-reading strategies to comprehension, reading time, and phonemic awareness. Reading Research Quarterly, 27, 141-151.

Cennamo, K. S., Savenye, W. C., \& Smith, P. L. (1991). Mental effort and video-based learning: The relationship of preconceptions and the effects of interactive and covert practice. Educational Technology Research and Development, $39(1), 5-16$

Choi, H. J., \& Johnson, S. D. (2005). The effect of context-based video instruction on learning and motivation in online courses. American Journal of Distance Education, 19(4), 215-227.

Dillenbourg, P., \& Traum, D. (2006). Sharing solutions: Persistence and grounding in multimodal collaborative problem solving. The Journal of the Learning Sciences, 15(1), 121-151.

Dowhower, S. L. (1987). Effects of repeated Reading on second-grade transitional readers' fluency and comprehension. Reading Research Quarterly, 22, 389-406.

Finn, J. D. (1989). Withdrawing from school. Review of Educational Research, 59(2), 117-142.

Giannakos, M. N. (2013). Exploring the video-based learning research: A review of the literature. British Journal of Educational Technology, 44(6), E191-E195.

Giannakos, M. N., Chorianopoulos, K., \& Chrisochoides, N. (2015). Making sense of video analytics: Lessons learned from clickstream interactions, attitudes, and learning outcome in a video-assisted course. The International Review of Research in Open and Distance Learning, 16(1), 260-283.

Good, T. L., \& Beckerman, T. M. (1978). Time on task: A naturalistic study in sixth-grade classrooms. The Elementary School Journal, 78(3), 193-201.

Grabe, M., \& Latta, R. M. (1981). Cumulative achievement in a mastery instructional system: The impact of differences in resultant achievement motivation and persistence. American Educational Research Journal, 18(1), 7-13.

Hastie. (1993). In chambers and Hastie (1993) statistical models in S. Chapman and Hall.

Hastie and Tibshirani. (1990). Generalized additive models. Chapman and Hall.

Holmqvist, K., Nyström, M., Andersson, R., Dewhurst, R., Jarodzka, H., \& Van de Weijer, J. Eye tracking: A comprehensive guide to methods and measures. OUP Oxford, 2011. 
Jermann, P., \& Nüssli, M. A. (2012). Effects of sharing text selections on gaze cross-recurrence and interaction quality in a pair programming task. In Proceedings of the ACM 2012 conference on computer supported cooperative work (pp. 1125-1134). ACM.

Just, M. A., \& Carpenter, P. A. (1980). A theory of reading: From eye fixations to comprehension. Psychological Review, 87(4), 329.

Kaplan, R. M. (1982). Nader's raid on the testing industry: Is it in the best interest of the consumer? The American Psychologist, $37(1), 15$.

Kim, J., Nguyen, P. T., Weir, S., Guo, P. J., Miller, R. C., \& Gajos, K. Z. (2014). Crowdsourcing step-by-step information extraction to enhance existing how-to videos. In Proceedings of the SIGCHI conference on human factors in computing systems (pp. 4017-4026). ACM.

Kizilcec, R. F., Papadopoulos, K., \& Sritanyaratana, L. (2014). Showing face in video instruction: Effects on information retention, visual attention, and affect. In Proceedings of the SIGCHI conference on human factors in computing systems (pp. 20952102). ACM.

Liaw, A., \& Wiener, M. (2002). Classification and regression by randomForest. $R$ news, 2(3), 18-22.

Mayer, R. E., \& Moreno, R. (2003). Nine ways to reduce cognitive load in multimedia learning. Educational Psychologist, 38(1), $43-52$.

Millis, K. K., \& King, A. (2001). Rereading strategically: The influences of comprehension ability and a prior reading on the memory for expository text. Reading Psychology, 22(1), 41-65.

Moreno-Marcos, P. M., Alario-Hoyos, C., Muñoz-Merino, P. J., \& Kloos, C. D. (2018). Prediction in MOOCs: A review and future research directions. IEEE Transactions on Learning Technologies, 12(3), 384-401.

Paivio, A. (2013). Imagery and verbal processes. Psychology Press.

Paris, S. G., \& Jacobs, J. E. (1984). The benefits of informed instruction for children's reading awareness and comprehension skills. Child development, 2083-2093.

Pelánek, R. (2015). Metrics for evaluation of student models. Journal of Educational Data Mining, 7(2), 1-19.

Prieto, L. P., Sharma, K., Dillenbourg, P., \& Jesús, M. (2016). Teaching analytics: Towards automatic extraction of orchestration graphs using wearable sensors. In Proceedings of the sixth international conference on Learning Analytics \& Knowledge (pp. 148-157). ACM.

Rasmussen, C. E. (2003). Gaussian processes in machine learning. In Summer School on machine learning (pp. 63-71). Berlin, Heidelberg: Springer.

Reinking, D. (1988). Computer-mediated text and comprehension differences: The role of Reading time, reader preference, and estimation of learning. Reading Research Quarterly, 23, 484-498.

Richardson, D. C., Dale, R., \& Kirkham, N. Z. (2007). The art of conversation is coordination. Psychological Science, 18(5), 407413.

Scholkopf, B., \& Smola, A. J. (2001). Learning with kernels: Support vector machines, regularization, optimization, and beyond. MIT press.

Schwartz, D. L., \& Hartman, K. (2007). It is not television anymore: Designing digital video for learning and assessment. Video Research in the Learning Sciences edited by Ricki Goldman, Roy Pea, Brigid Barron, Sharon J. Derry, 335-348.

Seaton, D. T., Bergner, Y., Chuang, I., Mitros, P., \& Pritchard, D. E. (2014). Who does what in a massive open online course?

Severin, W. (1967). Another look at cue summation. AV Communication Review, 15(3), 233-245.

Sharma, K., Caballero, D., Verma, H., Jermann, P., \& Dillenbourg, P. (2015a). Looking AT versus looking THROUGH: A dual eyetracking study in MOOC context. In the proc. of the Computer Supported Collaborative Learning 205 (pp. 260-267). International Society of the Learning Sciences, Inc.[ISLS].

Sharma, K., Caballero, D., Verma, H., Jermann, P., \& Dillenbourg, P. (2015b). Shaping learners' attention in massive open online courses. Revue internationale des technologies en pédagogie universitaire/International Journal of Technologies in Higher Education, 12(1-2), 52-61.

Sharma, K., Jermann, P., \& Dillenbourg, P. (2014). "With-me-ness": A gaze-measure for students' attention in MOOCs. In Proceedings of international conference of the learning sciences 2014 (no. CONF (pp. 1017-1022). ISLS.

Simonyan, K., \& Zisserman, A. (2014). Very deep convolutional networks for large-scale image recognition arXiv preprint arXiv: 1409.1556.

Špakov, O., \& Miniotas, D. (2007). Visualization of eye gaze data using heat maps. Elektronika ir elektrotechnika, 74, 55-58.

Tibshirani, R. (1996). Regression shrinkage and selection via the lasso. Journal of the Royal Statistical Society: Series B: Methodological, 58(1), 267-288.

Tobagi, F. A. (1995). Distance learning with digital video. IEEE Multimedia, 2(1), 90-93.

Volery, T., \& Lord, D. (2000). Critical success factors in online education. International Journal of Educational Management, $14(5), 216-223$.

Wiley, D. E. (1976). Another hour, another day: Quantity of schooling, a potent path for policy. In Schooling and achievement in American society (pp. 225-265).

\section{Publisher's Note}

Springer Nature remains neutral with regard to jurisdictional claims in published maps and institutional affiliations. 